the uranium was determined, with concordant results, by four different methods) has no visible inclusions. The total volume provided by fissures would be so small that it would be necessary to assume in them a concentration of uranium far greater than is, in fact, found in silicates or sulphides of meteoritic origin.

A definite test whether or not the radioactive material is concentrated along fissures could be obtained by photographic methods which, however, are difficult in this case owing to the very low activities. We are much indebted to Dr. E. Picciotto, of the Laboratoire de Physique Nucléaire at Brussels, for privately showing us preliminary results obtained by him and Mrs. Deutsch with a slice of the Carbo meteorite; these do not seem to us to lend any support to Prof. Urey's hypothesis ; but Dr. Picciotto hopes to increase the accuracy, and his final results will be awaited with great interest.

In the last paragraph but one of his article, Prof. Urey refers in four or five consecutive sentences to as many possibilities for the origin and age of meteorites. We would like to suggest that our views should be added as one more possibility.

Max-Planck-Institut für Chemie

$$
\text { F. A. Paneth }
$$

Mainz.

G. R. Martin

S. J. THOMSON

Londonderry Laboratory for Radiochemistry, University of Durham.

${ }^{1}$ Nature, 175, 321 (1955).

\section{"Applied Statistics"}

IN his roview of my book "Statistical Methods in Electrical Engineering" (Nature, March 19, p. 484), Mr. F. Downton criticizes my statement that Bayes theorem is controversial. The heading of the paragraph in Sir Ronald Fisher's book which I cited is "The Rejection of Inverse Probability". Coupled with the lack of mention of Bayes beyond the introductory chapter, I can only take this to mean that the author did not accept the rigorous validity of anything which Bayes formulated. To me, therefore, it appears that Mr. Downton's statement is demonstrably untrue. Faced with this controversial issue when writing a practical book, I decided to mention the existence of the controversy and then take a firm decision (the opposito of Fisher's) which was justified primarily on empirical grounds.

The question of $\sqrt{ }(N-1)$ depends on a distinction between $(a)$ the disporsion of a group of observations and $(b)$ the probable difference between the mean of the group and a determinate true value.

If my book should survivo the disfavour of statisticians and roach a second edition, I will bear in mind Mr. Downton's suggestion that congestion theory should be mentioned, though I should not attempt to satisfy his desire for a "definitive" treatment of this subject.

Electrical Engineering Department,

D. A. Berra

University of Birmingham.

ThE position with regard to Bayes' theorem is admirably set out in M. G. Kendall's "The Advanced Theory of Statistics", Vol. 1, pp. 175-176 (3rd edit., 1947), where my statement that it is the assumptions and not the theorem which are controversial is sup- ported. Reconciliation of the quotations from Fisher, Jeffreys and my review, which Dr. Bell finds contradictory, depends on the recognition that the terms 'postulate', 'axiom' and 'theorem' are not synonymous.

The extract from Dr. Bell's book, which I quoted in my review, makes it quite clear that he stated, erroneously, that the standard deviation of the mean of $N$ observations varies inversely as $\sqrt{N-1}$ (that is, presumably, case (b) above); for the correct expression see any elementary text-book; for example, C. E. Weatherburn, "A First Course in Mathematical Statistics", p. 185 (1946). It is to be hoped that this correspondence will not give readers the impression that these are matters which are likely to cause disagreement among statisticians.

Department of Applied Mathematics, F. Downton

University of Liverpool.

\section{An Amplifier based on the Hall Effect}

THE achievement of a Hall effect amplifier by Ross and Thompson, as recorded in Nature of March 19 , p. 518 , is quite remarkable, even taking into consideration the very high carrier mobility obtainable with indium antimonide. Calculation shows that a power gain is only possible if every effort is made to get the required magnetic field with the minimum power input, and this clearly entails the use. of a high permeability core with a very thin crystal in a correspondingly small air-gap. The power gain is derived from the input of current to the crystal, and theoretically there appears to be no limit to the amplification obtainable if it were possible to raise the strength of the magnetic field indefinitely. In practice, saturation must occur. By the same argument an input signal applied to the current terminals of the crystal when immersed in a steady magnetic field could not lead to amplification. There is then no independent source of power input, and in this case the usual expression for the Hall output cannot therefore remain valid with large signals. The amplifier of Ross and Thompson seems to be limited to low-frequency applications unless a semi-conductor of still higher carrier mobility becomes available.

It should be possible to make the amplifier into an oscillator by feeding back the output from the Hall terminals to the magnetizing coil.

\section{University College, \\ London, W.C.1.}

H. M. BarLow

We agree with Prof. Barlow that considerable refinement in technique is required to achieve gain. In addition to those points which he mentions as essential, it is necessary also to have very good thermal contact between the crystal and the magnetic core, in order that a high input current density may be used without appreciable heating of the crystal. Although only small power gains have so far been achieved, we think that the device is capable of considerable development.

I. M. Ross

N. A. C. ThоMPson

Services Electronics Research Laboratory, Baldock, Herts. 